\title{
Role of Neoadjuvant Chemotherapy (NACT) Followed by Surgical Cytoreduction in Advanced Epithelial Ovarian Cancer
}

\author{
Batra Sonia $\cdot$ Nayak Himanshu $\cdot$ Dave Kalpna S.
}

Received: 18 August 2011/Accepted: 5 November 2011/Published online: 7 March 2012

(C) Federation of Obstetric \& Gynecological Societies of India 2012

\begin{abstract}
Objectives To study the role of neoadjuvant chemotherapy (NACT) followed by surgical cytoreduction in the management of advanced epithelial ovarian cancers.

Methods A prospective hospital based study of patients with advanced epithelial ovarian cancers (stage III and IV) was conducted at Gujarat Cancer \& Research Institute, Ahmedabad during August 2008 to August 2010. Total 50 patients were treated with NACT followed by surgical cytoreduction and followed up till August 2010. Response to NACT, optimal cytoreduction rate and overall response rate were analyzed.

Results There were 43 patients $(86 \%)$ with stage III disease and 7 (14\%) with stage IV disease. All patients were given NACT and after NACT, complete response occurred in 17 patients (34\%), 27 (54\%) had partial response. Optimal surgical cytoreduction could be achieved in $36(72 \%)$ of the patients. The median follow up was 19 months.
\end{abstract}

Batra S., Resident · Dave K. S., Professor \& Head

Department of Obstetrics \& Gynecology, Gujarat Cancer

Research Institute, Ahmedabad 380 016, India

Nayak H., Assistant Professor

Department of Community Medicine, AMC MET Medical College,

Ahmedabad 380008, India

Batra S. $(\bowtie)$, Resident

244, APR Colony, Katanga, Jabalpur 482001, Madhya Pradesh, India e-mail: sanyo9992001@yahoo.com
Conclusions NACT followed by surgical cytoreduction is a promising treatment strategy for the management of advanced epithelial ovarian cancers.

Keywords Advanced epithelial ovarian cancer . Neoadjuvant chemotherapy $\cdot$ Surgical cytoreduction

\section{Introduction}

Ovarian cancer is the second most common gynecological malignancy after cervical cancer and is the fourth most common cause of death from malignancy in women [1,2]. It is the fifth most common cancer in women in the United States. It accounts for $4 \%$ of all female cancers, $31 \%$ of cancers of the female genital tract and $5 \%$ of estimated cancer deaths [3].

A woman's risk at birth of having ovarian cancer some time in her lifetime is nearly $1.5 \%$, and the risk of dying is almost $1 \%$. The death rate per 100,000 has decreased by $8 \%$ from 9.51 in 1991 to 8.75 in 2004 . In India the prevalence is $10 / 100,000$.

Epithelial ovarian cancer, consists $90 \%$ of all ovarian cancers, has the highest case fatality ratio because more than two-thirds of patients have advanced disease at diagnosis (stage III and IV classified by International federation of gynecology \& obstetrics) [4]. Advanced ovarian cancer is a disease associated with poor prognosis, with the 5 year survival ranging from 5 to $25 \%[1,5,6]$. It presents a major surgical challenge, requires intensive and often complex 
therapies, and is extremely demanding of the patient's psychological and physical energy.

Primary cytoreductive surgery followed by platinum plus taxol based chemotherapy is currently the standard treatment for patients with advanced stage III and IV. Studies have shown that by primary cytoreductive surgery about $30-70 \%$ of patients with advanced disease are not optimally debulked [7]. Patients with poor performance status and those with lesion involving diaphragm, base of small bowel, mesentery, liver parenchyma, retroperitoneal nodes and upper abdominal structures, stomach and splenic hilum are often unresectable, otherwise even if resectable it requires major surgical intervention in the form of bowel resection anastomoses, colostomy, ileostomy etc. leading to further physical morbidity and mental stress, depression, lack of confidence and lack of social acceptability on the part of the patient.

Ovarian cancer is a chemo-sensitive tumor and majority of the patients receive adjuvant chemotherapy. Recently, neoadjuvant chemotherapy (NACT) has been advocated for patients with advanced ovarian cancer with an aim to improve resectability rates and survival [8]. In view of this many investigators have used primary or upfront NACT as an alternative to primary surgery with the goal of improving surgical quality. Rational behind using NACT is to make advanced inoperable disease to operable, increased optimal debulking rate and reduced post operative morbidity as compared to primary surgery. Hence the following study has been conducted to describe the socio-demographic profile and pattern of presentation of ovarian cancer patients and to evaluate the role of NACT in advanced epithelial ovarian cancer which is clinically inoperable on presentation, with respect to response rate, resectibility rate, extent of resection during interval debulking surgery, whether optimal or suboptimal cytoreduction.

\section{Methods}

A prospective hospital based study of advanced ovarian cancer (stage III and IV) was conducted at the Department of Gynecologic Oncology, G.C. \& R.I., Ahmedabad. Total 50 patients of advanced epithelial ovarian cancer stage III and IV were included from August 2008 to August 2010, period of 2 years. All these patients were followed up till August 2010.

All patients with stage IV disease and stage IIIC with large volume ascites $(>500 \mathrm{ml})$, extensive peritoneal disease and CA125>500, not amenable for optimal cytoreduction and patients considered unresectable by the treating surgical team were subjected to NACT followed by surgical cytoreduction after obtaining an informed consent. Patients who had significant primary surgical cytoreduction (any cytoreductive procedure other than exploratory laparotomy and biopsy) elsewhere were excluded from the study.

On presentation each patient was evaluated thoroughly by a detailed history regarding symptoms, past and family history. A thorough physical examination including Breast, neck, per abdomen, per speculum, per vaginal, per rectal examinations to assess ovarian mass, ascites, hepatospleenomegaly, peritoneal disease, pleural effusion, supraclavicular lymph nodes, was performed. Radiological examination either USG pelvis or CT scan and Chest X-ray to evaluate the extent of disease, involvement and to decide staging and unresectability. Serum CA 125 level was measured in every patient on presentation and graded whether $<500$ or $>500$ to assess the response to NACT and for prognosis. Cytological or histopathological confirmation (USG guided biopsy from the ovarian mass) was done in each case before starting NACT.

NACT was started after establishing histopathological diagnosis and clinical staging (being inoperable).

The commonest combination used was cisplatin + cyclophosphamide (Arm A) or paclitaxel + carboplatin (Arm B) every 21 days. Each patient was monitored for chemotherapy related adverse reaction graded according to WHO criteria.

Response evaluation was done after $3 \mathrm{rd} / 4$ th cycle of NACT and if there was no disease progression or tumor was responding, and if the disease was found clinically and radiologically resectable, patients were taken for interval cytoreductive surgery.

Standard debulking procedure includes total abdominal hysterectomy + bilateral salpingo oophorectomy + infra colic omentectomy and bilateral lymphnode dissection (or other metastasis as required) to achieve complete or optimal or suboptimal resection. Response of NACT was also assessed by extent of surgical resection possible (whether optimal or suboptimal debulking done).

After completion of postoperative adjuvant chemotherapy cycles, patients were followed up every 3 monthly by clinical and radiological examination and CA 125 levels (if indicated) to detect local or systemic failure. Patients with recurrence were offered salvage chemotherapy. Patients were categorized as below:

(1) Complete remission (CR) — no clinical and radiological evidence of disease.

(2) Partial remission (PR) measurable tumor mass has decreased by $50 \%$ and there are no new areas of tumor developing after treatment as well as no areas of tumor shows progression.

(3) Overall remission rate (ORR) - CR + PR.

(4) Minimal response-same as partial response, but degree of response is less than $50 \%$. 
(5) Progression- the tumor mass has increased more than $25 \%$ at one or more sites or new lesions have appeared.

(6) Stable disease (SD) - measurable disease has remained the same and does not meet the criteria for CR, PR, minimal response or progression.

(7) Optimal debulking surgery—residual tumor $<0.5 \mathrm{~cm}$ in maximum diameter [9].

(8) Suboptimal debulking surgery-residual tumor $\geq 2 \mathrm{~cm}$ in maximum diameter.

(9) Prognosis free survival (PFS) from documented no evidence of disease (NED) at end of complete treatment to first sign of recurrence (radiology/rising CA-125 level).

Data was collected and compiled in the excel sheet and analyzed with the appropriate statistical tests.

\section{Results}

Total 50 patients were included in our study with advanced stage ovarian carcinoma who presented to our institute between August 2008 and August 2010.

Majority of patients 22(44\%) were in age group of $40-50$ years while only $3(6 \%)$ were among the $60-70$ years. Mean age of patients was $50.14 \pm 8.2$ years. $78 \%$ patients were postmenopausal, $16 \%$ were premenopausal and $6 \%$ of the patients had surgical menopause. $90 \%$ of the patients were multiparous whereas $6 \%$ were nulliparous and $4 \%$ primiparous.

Abdominal distension plus abdominal pain was the most common symptom amongst the $18(36 \%)$ patients followed by abdominal pain alone amongst $15(30 \%)$ of patients while only $4(8 \%)$ of the patients had bladder or bowel complaints along with abdominal distension.

Ascites was the commonest $37(74 \%)$ clinical finding while mass was palpable in 15(30\%) and clinically palpable omental cake was found in $5(10 \%)$ patients whereas $6(12 \%)$ had no significant per abdomen findings. On per vaginal examination, forniceal fullness was found in $18(36 \%)$ of the patients while mass was palpable in $31(62 \%)$ of the patients and $1(2 \%)$ patient had no abnormal finding. Nodularity was found in pouch of doughlas on per rectal examination in $36(72 \%)$ of the patients.

Pleural effusion was found in only $9(18 \%)$ while maximum $41(82 \%)$ of the patients had normal chest X-ray.

Maximum 37(74\%) patients had CA 125 levels $>500$ on presentation followed by $13(26 \%)$ had CA 125 level 101-500 while none of the patients had baseline CA125 in the normal range. Range of baseline CA 125 was 164-5394.

$31(62 \%)$ of the patients had pelvis as well as per abdomen disease on USG while $16(32 \%)$ had disease only in the pelvis and $3(6 \%)$ patients had only per abdomen disease that is ascites/liver/peritoneal metastasis with no evidence of pelvic mass on USG.

Out of 26 patients with ascites, 21(81\%) patients had positive ascitic fluid cytology for malignant cells while $5(55 \%)$ patients had pleural fluid cytology positive amongst 9 patients with pleural effusion.

There were 15 patients in Arm A and 35 patients in Arm B. Out of the total 50 patients, Majority $43(86 \%)$ patients had stage III disease while only $7(14 \%)$ were stage IV disease with 5 patients having positive pleural fluid cytology and 2 patients with liver metastasis. Mean number of neoadjuvant CT cycles given were 3 in both Arm A (66\%) and in Arm B (71\%).

After NACT 17(34\%) patients had NED on USG while $27(54 \%)$ had disease confined to pelvis only.

Table 1 shows disease characteristics on USG before and after NACT. Out of 3(6\%) patients who had only per abdomen disease previously, depicted total regression of disease after NACT showing $100 \%$ response. Pleural effusion and ascites resolved in all the patients after NACT. Hence there was significant reduction of disease with $P$ value $<0.0001$. There was statistically significant reduction of disease found between pelvis and P/A disease $(P<0.0001)$ and between $\mathrm{P} / \mathrm{A}$ and pelvis $+\mathrm{P} / \mathrm{A}(P<$ $0.0001)$.

Out of 50 patients, 22(44\%) patients had CA 125 values within the normal range $(<35)$ after NACT while $23(46 \%)$ had values between 35 and 100 with only one patient (2\%) had CA $125>500$ as compared to baseline CA 125 values where $37(74 \%)$ had $>500$. Thus, statistically significant

Table 1 Comparison of USG findings before and after NACT

\begin{tabular}{lcccccc}
\hline Basal USG characteristics & \multicolumn{2}{l}{ After NACT-USG/CT SCAN characteristics } & & Total & $Z$ value & $P$ value \\
\cline { 2 - 4 } & NAD $(\%)$ & Pelvis $(\%)$ & Pelvis + P/A (\%) & & \\
\hline Abdomen disease & $3(100)$ & $0(0.0)$ & $0(0.0)$ & $3(6 \%)$ & 1 & 1 \\
Pelvis disease & $6(37.5)$ & $9(56.3)$ & $1(6.2)$ & $16(32 \%)$ & 5.16 \\
Pelvis + abdomen & $8(25.8)$ & $18(58.1)$ & $5(16.1)$ & $31(62 \%)$ & 9.44 \\
Total & $17(34)$ & $27(54)$ & $6(12)$ & $50(100)$ & $<0.0001$ \\
\hline
\end{tabular}


difference $(Z=6.154, P<0.0001)$ was found between CA 125 level before and after NACT.

Overall response rate to NACT was $80 \%$ in Arm A while it was $91 \%$ in Arm B. Combining the two arms the ORR of study group was $88 \%$ (Table 2 ).

Majority $36(72 \%)$ of the patients underwent optimal cytoreduction with residual disease $<0.5 \mathrm{~cm}$ while only $14(28 \%)$ of patients underwent suboptimal cytoreduction with residual disease $>2 \mathrm{~cm}$ (Table 3 ).

Out of the 17 patients with CR, 11(65\%) underwent optimal cytoreduction while out of 27 patients with PR, $22(81 \%)$ underwent optimal cytoreduction. Out of the 6 patients with SD, 3(50\%) underwent optimal cytoreduction and $3(50 \%)$ underwent suboptimal cytoreduction.

On histopathology, 46 had serous papillary cystadenocarcinoma, 2 patients had endometroid adenocarcinoma and 2 had mucinous adenocarcinoma. 26 patients had Grade III carcinoma (Table 4).

After surgery, 31(62\%) patients had CR at the end of treatment and were evaluable with a median follow up of 19 months. 3 patients $(6 \%)$ had gross residual disease at the end of treatment called the primary refractory disease. 1 patient died due to disease. 5 patients were lost to follow up. 2 patients $(1+1 \mathrm{LFU})$ had recurrence with a median PFS of 3.5 months.

\section{Discussion}

Surgical debulking followed by platinum based chemotherapy is the standard of care for patients with advanced stage ovarian cancer. NACT followed by interval debul-

Table 2 Distribution of NACT response according to chemotherapy

\begin{tabular}{llcr}
\hline Response & \multicolumn{2}{l}{ Chemotherapy } & Total \\
\cline { 2 - 3 } & Arm A (\%) & Arm B (\%) & \\
\hline CR & $4(26.7)$ & $13(37.1)$ & $17(34)$ \\
PR & $8(53.3)$ & $19(54.3)$ & $27(54)$ \\
SD & $3(20)$ & $3(8.6)$ & $6(12)$ \\
Total & $15(100)$ & $35(100)$ & $50(100)$ \\
\hline
\end{tabular}

Table 3 Interval debulking surgery pattern among patients

\begin{tabular}{llll}
\hline Interval surgery & Chemo & Total \\
\cline { 2 - 3 } & Arm A (\%) & Arm B (\%) & \\
\hline $\begin{array}{l}\text { Optimal cytoreduction } \\
(\text { Residual disease }<0.5 \mathrm{~cm})\end{array}$ & $11(73.3)$ & $25(71.4)$ & $36(72)$ \\
$\begin{array}{l}\text { Suboptimal cytoreduction } \\
\quad(\text { RES DIS }>2 \mathrm{~cm})\end{array}$ & $4(26.7)$ & $10(28.6)$ & $14(28)$ \\
\begin{tabular}{l} 
Total \\
\hline
\end{tabular} & $15(100)$ & $35(100)$ & $50(100)$ \\
\hline
\end{tabular}

Table 4 Histo-pathological examination (HPE) characteristics of ovarian mass

\begin{tabular}{lccc}
\hline HPE type & \multicolumn{2}{r}{ Chemo } & Total (\%) \\
\cline { 2 - 3 } & Arm A (\%) & Arm B (\%) & \\
\hline Mucinous adenocarcinoma & $0(0)$ & $2(5.7)$ & $2(4)$ \\
Endometroid adenocarcinoma & $0(0)$ & $2(5.7)$ & $2(4)$ \\
Serous papillary & $15(100)$ & $31(88.6)$ & $46(92)$ \\
$\quad$ cystadenocarcinoma & & & \\
Grade I & $2(13.3)$ & $9(25.7)$ & $11(22)$ \\
Grade II & $4(26.7)$ & $9(25.7)$ & $13(26)$ \\
Grade III & $9(60)$ & $17(48.6)$ & $26(52)$ \\
Total & $15(100)$ & $35(100)$ & $50(100)$ \\
\hline
\end{tabular}

king may reduce the tumor burden and allow for an easier and complete surgical cytoreduction, at least in patients with chemo sensitive disease.

As we know, there is an inverse relationship between residual tumor diameter after surgery and survival [10]. Prognosis largely depends upon the degree of cytoreduction achieved during primary surgery. Gross residual disease more than $2 \mathrm{~cm}$ carries poor prognosis, shown in the meta-analysis by Bristow et al. [10]. Most people today accept optimal cytoreduction as residual tumor volume of $<1 \mathrm{~cm}$ or even $<0.5 \mathrm{~cm}$. However, due to bulky disease in advanced ovarian cancer, optimal cytoreduction is possible in only $40-50 \%$ of the patients [7]. These data suggest that patients with advanced stage ovarian cancer could only benefit from an optimal surgical debulking, which usually does not exceed $40 \%$ even in experienced surgical centers in case of primary debulking procedure. Hence NACT may be helpful in achieving cytoreduction and converting possible suboptimal initial surgery into optimal surgery. Several studies [8, 9, 11-15] have established the role of platinum based NACT in advanced stage ovarian cancer without compromising OS or PFS and offering better quality of life.

In this study we have evaluated the role of NACT in the management of advanced stage epithelial ovarian tumors in various aspects.

Maximum number of patients 22(44\%) were in the age group 40-50 years in the study. $78 \%$ were postmenopausal women in the study which correlate with the study of Devita Jr. et al. [5]. The commonest symptom was abdominal pain and distension and commonest finding was ascites in $74 \%$ of total patients. This sign-symptom was almost equal in both the arms and also correlates with the study of Devita Jr. et al. [5].

In radiological findings, 31 patients had pelvis and upper abdominal distension and only $6 \%$ of patients had only per abdominal distension and all patients measurable tumor 
masses of $>2 \mathrm{~cm}$ before NACT correlating with study of Le et al. [9].

$86 \%$ of patients were in stage III in our study, similar to Le et al. [9].

Mean number of NACT cycles in both arms were 3. $10(66 \%)$ patients in Arm A and 25(71.4\%) patients in Arm $\mathrm{B}$ were given 3 cycles. More number of patients got response with 3 cycles in Arm B as compared to Arm A.

ORR was $88 \%$ with CR of $34 \%$ and PR of $54 \%$, which was similar to findings by Mazzeo et al. [8], Le et al. [9], Chan et al. [13], Ansqucr et al. [14].

This result shows that response to paclitaxel plus carboplatin is significantly higher despite having more number of patients with more extensive disease and lesser number of mean neoadjuvant cycles as previously seen.

We achieved optimal cytoreduction $(<2 \mathrm{~cm})$ after NACT in $72 \%$ of patients which was similar to Mazzeo et al. [8] (69\%), Le et al. [9] (80\%), Chan et al. [13] (77\%), Ansquer et al. [14] (72\%). Our study had only 52\% of patients having grade III as compared to other studies which have greater patients in grade III. This explains high rate of optimal debulking surgery in our study group. Significant reduction in CA125 was found similar to findings of Le et al. [9, 16]. Overall 14(28\%) had suboptimal and $36(72 \%)$ had optimal debulking surgery in the study. Overall $62 \%$ of the patients had NED at the end of study. Median follow up was found to be 19 months, while median progression free survival was 3.5 months and median survival of 13 months.

\section{Conclusion}

Patients with advanced ovarian carcinoma of FIGO Stage III onwards at diagnosis are at high risk for surgery as radical surgeries may be required and it is associated with considerable intra and post operative morbidity and moreover benefit only marginally from conventional therapy with more of suboptimal debulking rather than optimal debulking. Such patients can be benefited with primary NACT followed by debulking surgery with goal of improving surgical quality and increasing the chance of optimal debulking $<0.5 \mathrm{~cm}$ residual disease.

The results of the current study show that NACT is a reasonable and promising approach for the management of advanced ovarian cancer. A significant number of patients respond to NACT leading to a higher optimal surgical cytoreduction, which may result in improved survival.

\section{References}

1. Yeole BB, Kumar AV, Kurkure A, et al. Population-based survival from cancers of breast, cervix and ovary in women in Mumbai, India. Asian Pac J Cancer Prev. 2004;5:308-15.

2. Bray F, Loos AH, Tognazzo S, et al. Ovarian cancer in Europe: cross-sectional trends in incidence and mortality in 28 countries, 1953-2000. Int J Cancer. 2005;113:977-90.

3. Greenlee RT, Hill-Harmon MB, Murray T, et al. Cancer statistics, 2001. CA Cancer J Clin. 2001;15:15-36.

4. Jemal A, Murray T, Samuels A, et al. Cancer statistics, 2003. CA Cancer J Clin. 2003;53:5-26.

5. Ozols RF, Schwartz PE, Eifel PJ. Ovarian cancer, fallopian tube carcinoma and peritoneal carcinoma. In: DeVita Jr VT, Hellman S, Rosenberg SA, editors. Cancer: principles and practice of oncology. 6th ed. Lippincott: Williams and Wilkins; 2001. p. $1597-632$.

6. Beard CM, Hartmann LC, Atkinson EJ, et al. The epidemiology of ovarian cancer: a population-based study in Olmsted County, Minnesota, 1935-1991. Ann Epidemiol. 2000;10:14-23.

7. van der Burg ME. Advanced ovarian cancer. Curr Treat Options Oncol. 2001;2(2):109-18.

8. Mazzeo F, Berliere M, Kerger J, et al. Neoadjuvant chemotherapy followed by surgery and adjuvant chemotherapy in patients with primarily unresectable, advanced-stage ovarian cancer. Gynecol Oncol. 2003;90:163-9.

9. Le T, Faught W, Hopkins L, et al. Primary chemotherapy and adjuvant tumor debulking in the management of advanced stage epithelial ovarian cancers. Int J Gynecol Cancer. 2005; 15(5):770-5.

10. Bristow RE, Tomacruz RS, Armstrong DK, et al. Survival effect of maximal cytoreductive surgery for advanced ovarian carcinoma during the platinum era: a meta-analysis. J Clin Oncol. 2002;20:1248-59.

11. Patel S, Desai A, Chauhan A, et al. Single agent carboplatin as neoadjuvant chemotherapy in advanced epithelial ovarian cancer. Indian J Gynecol Oncol. 2009;9(1):51-6.

12. Hou JY, Kelly MG, Yu H, et al. Neoadjuvant chemotherapy lessens surgical morbidity in advanced ovarian cancer and leads to improved survival in stage IV disease. Gynecol Oncol. 2007;105(1):211-7.

13. Chan YM, Ng TY, Ngan HYS, et al. Quality of life in women treated with NACT for advanced ovarian cancer: a prospective longitudinal study. Gynecol Oncol. 2003;88:9-16.

14. Ansquer Y, Leblanc E, Clough K, et al. Neoadjuvant chemotherapy for unresectable ovarian carcinoma. A French multicenter study. Cancer. 2001;91:2329-34.

15. Kuhn W, Rutke S, Spathe K, et al. Neoadjuvant chemotherapy followed by tumor debulking prolongs survival for patients with poor prognosis in FIGO stage IIIc ovarian carcinoma. Cancer. 2001;92:2585-91.

16. Le T, Williams K, Senterman M, et al. Histopathologic assessment of chemotherapy effects in epithelial ovarian cancer patients treated with NACT \& delayed primary surgical debulking. Gynecol Oncol. 2007;106:160-3. 